\title{
Adaptive Walks on Changing Landscapes: Levins' Approach Extended
}

C. Rueffler (rueffler@ rulsfb.leidenuniv.nl)

T.J.M. Van Dooren (vdooren @ rulsfb.leidenuniv.nl)

J.A.J. Metz (metz@ rulsfb.leidenuniv.nl)

\section{Approved by}

Ulf Dieckmann

Program Leader, Adaptive Dynamics Network

December 2004 Institute, its National Member Organizations, or other organizations supporting the work. 


\section{IIASA STUdIES IN ADAPTIVE DYNAMICS No. 89}

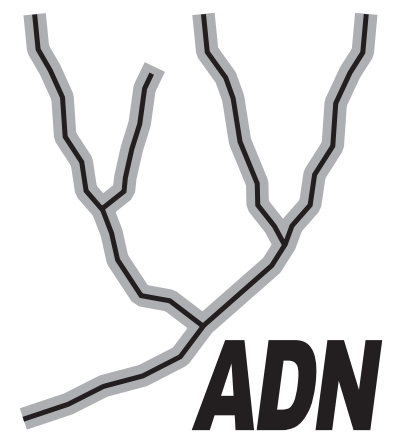

The Adaptive Dynamics Network at IIASA fosters the development of new mathematical and conceptual techniques for understanding the evolution of complex adaptive systems.

Focusing on these long-term implications of adaptive processes in systems of limited growth, the Adaptive Dynamics Network brings together scientists and institutions from around the world with IIASA acting as the central node.

Scientific progress within the network is collected in the IIASA Studies in Adaptive Dynamics series.

No. 1 Metz JAJ, Geritz SAH, Meszéna G, Jacobs FJA, van Heerwaarden JS: Adaptive Dynamics: A Geometrical Study of the Consequences of Nearly Faithful Reproduction. IIASA Working Paper WP-95-099 (1995). van Strien SJ, Verduyn Lunel SM (eds): Stochastic and Spatial Structures of Dynamical Systems, Proceedings of the Royal Dutch Academy of Science (KNAW Verhandelingen), North Holland, Amsterdam, pp. 183-231 (1996).

No. 2 Dieckmann U, Law R: The Dynamical Theory of Coevolution: A Derivation from Stochastic Ecological Processes. IIASA Working Paper WP-96-001 (1996). Journal of Mathematical Biology 34:579-612 (1996).

No. 3 Dieckmann U, Marrow P, Law R: Evolutionary Cycling of Predator-PreyInteractions: Population Dynamics and the Red Queen. IIASA Preprint (1995). Journal of Theoretical Biology 176:91-102 (1995).

No. 4 Marrow P, Dieckmann U, Law R: Evolutionary Dynamics of Predator-Prey Systems: An Ecological Perspective. IIASA Working Paper WP-96-002 (1996). Journal of Mathematical Biology 34:556-578 (1996).

No. 5 Law R, Marrow P, Dieckmann U: On Evolution under Asymmetric Competition. IIASA Working Paper WP-96-003 (1996). Evolutionary Ecology 11:485-501 (1997).

No. 6 Metz JAJ, Mylius SD, Diekmann O: When Does Evolution Optimize? On the Relation Between Types of Density Dependence and Evolutionarily Stable Life History Parameters. IIASA Working Paper WP-96-004 (1996).

No. 7 Ferrière R, Gatto M: Lyapunov Exponents and the Mathematics of Invasion in Oscillatory or Chaotic Populations. Theoretical Population Biology 48:126-171 (1995).

No. 8 Ferrière R, Fox GA: Chaos and Evolution. IIASA Preprint (1996). Trends in Ecology and Evolution 10:480485 (1995).

No. 9 Ferrière R, Michod RE: The Evolution of Cooperation in Spatially Heterogeneous Populations. IIASA Working Paper WP-96-029 (1996). The American Naturalist 147:692717 (1996).

No. 10 van Dooren TJM, Metz JAJ: Delayed Maturation in Temporally Structured Populations with Non-Equilibrium Dynamics. IIASA Working Paper WP-96-070 (1996). Journal of Evolutionary Biology 11:41-62 (1998).
No. 11 Geritz SAH, Metz JAJ, Kisdi É, Meszéna G: The Dynamics of Adaptation and Evolutionary Branching. IIASA Working Paper WP-96-077 (1996). Physical Review Letters 78:2024-2027 (1997).

No. 12 Geritz SAH, Kisdi É, Meszéna G, Metz JAJ: Evolutionary Singular Strategies and the Adaptive Growth and Branching of the Evolutionary Tree. IIASA Working Paper WP-96-114 (1996). Evolutionary Ecology 12:35-57 (1998).

No. 13 Heino M, Metz JAJ, Kaitala V: Evolution of Mixed Maturation Strategies in Semelparous Life-Histories: The Crucial Role of Dimensionality of Feedback Environment. IIASA Working Paper WP-96-126 (1996). Philosophical Transactions of the Royal Society of London Series B 352:1647-1655 (1997).

No. 14 Dieckmann U: Can Adaptive Dynamics Invade? IIASA Working Paper WP-96-152 (1996). Trends in Ecology and Evolution 12:128-131 (1997).

No. 15 Meszéna G, Czibula I, Geritz SAH: Adaptive Dynamics in a 2-Patch Environment: A Simple Model for Allopatric and Parapatric Speciation. IIASA Interim Report IR-97-001 (1997). Journal of Biological Systems 5:265-284 (1997).

No. 16 Heino M, Metz JAJ, Kaitala V: The Enigma of Frequency-Dependent Selection. IIASA Interim Report IR97-061 (1997). Trends in Ecology and Evolution 13:367-370 (1998).

No. 17 Heino M: Management of Evolving Fish Stocks. IIASA Interim Report IR-97-062 (1997). Canadian Journal of Fisheries and Aquatic Sciences 55:1971-1982 (1998).

No. 18 Heino M: Evolution of Mixed Reproductive Strategies in Simple Life-History Models. IIASA Interim Report IR-97063 (1997).

No. 19 Geritz SAH, van der Meijden E, Metz JAJ: Evolutionary Dynamics of Seed Size and Seedling Competitive Ability. IIASA Interim Report IR-97-071 (1997). Theoretical Population Biology 55:324-343 (1999).

No. 20 Galis F, Metz JAJ: Why Are There So Many Cichlid Species? On the Interplay of Speciation and Adaptive Radiation. IIASA Interim Report IR-97-072 (1997). Trends in Ecology and Evolution 13:1-2 (1998). 
No. 21 Boerlijst MC, Nowak MA, Sigmund K: Equal Pay for all Prisoners/ The Logic of Contrition. IIASA Interim Report IR-97-073 (1997). American Mathematical Society Monthly 104:303-307 (1997). Journal of Theoretical Biology 185:281-293 (1997).

No. 22 Law R, Dieckmann U: Symbiosis Without Mutualism and the Merger of Lineages in Evolution. IIASA Interim Report IR-97-074 (1997). Proceedings of the Royal Society of London Series B 265:1245-1253 (1998).

No. 23 Klinkhamer PGL, de Jong TJ, Metz JAJ: Sex and Size in Cosexual Plants. IIASA Interim Report IR-97-078 (1997). Trends in Ecology and Evolution 12:260-265 (1997).

No. 24 Fontana W, Schuster P: Shaping Space: The Possible and the Attainable in RNA Genotype-Phenotype Mapping. IIASA Interim Report IR-98-004 (1998). Journal of Theoretical Biology 194:491-515 (1998).

No. 25 Kisdi É, Geritz SAH: Adaptive Dynamics in Allele Space: Evolution of Genetic Polymorphism by Small Mutations in a Heterogeneous Environment. IIASA Interim Report IR-98-038 (1998). Evolution 53:993-1008 (1999).

No. 26 Fontana W, Schuster P: Continuity in Evolution: On the Nature of Transitions. IIASA Interim Report IR-98-039 (1998). Science 280:1451-1455 (1998).

No. 27 Nowak MA, Sigmund K: Evolution of Indirect Reciprocity by Image Scoring/ The Dynamics of Indirect Reciprocity. IIASA Interim Report IR-98-040 (1998). Nature 393:573-577 (1998). Journal of Theoretical Biology 194:561574 (1998).

No. 28 Kisdi É: Evolutionary Branching Under Asymmetric Competition. IIASA Interim Report IR-98-045 (1998). Journal of Theoretical Biology 197:149-162 (1999).

No. 29 Berger U: Best Response Adaptation for Role Games. IIASA Interim Report IR-98-086 (1998).

No. 30 van Dooren TJM: The Evolutionary Ecology of Dominance-Recessivity. IIASA Interim Report IR-98-096 (1998). Journal of Theoretical Biology 198:519-532 (1999).

No. 31 Dieckmann U, O'Hara B, Weisser W: The Evolutionary Ecology of Dispersal. IIASA Interim Report IR-98-108 (1998). Trends in Ecology and Evolution 14:88-90 (1999).

No. 32 Sigmund K: Complex Adaptive Systems and the Evolution of Reciprocation. IIASA Interim Report IR-98-100 (1998). Ecosystems 1:444-448 (1998).

No. 33 Posch M, Pichler A, Sigmund K: The Efficiency of Adapting Aspiration Levels. IIASA Interim Report IR-98103 (1998). Proceedings of the Royal Society London Series B 266:1427-1435 (1999).

No. 34 Mathias A, Kisdi É: Evolutionary Branching and Coexistence of Germination Strategies. IIASA Interim Report IR-99-014 (1999).

No. 35 Dieckmann U, Doebeli M: On the Origin of Species by Sympatric Speciation. IIASA Interim Report IR-99-013 (1999). Nature 400:354-357 (1999).

No. 36 Metz JAJ, Gyllenberg M: How Should We Define Fitness in Structured Metapopulation Models? Including an Application to the Calculation of Evolutionarily Stable Dispersal Strategies. IIASA Interim Report IR-99-019 (1999). Proceedings of the Royal Society of London Series B 268:499508 (2001)
No. 37 Gyllenberg M, Metz JAJ: On Fitness in Structured Metapopulations. IIASA Interim Report IR-99-037 (1999). Journal of Mathematical Biology 43:545-560 (2001).

No. 38 Meszéna G, Metz JAJ: Species Diversity and Population Regulation: The Importance of Environmental Feedback Dimensionality. IIASA Interim Report IR-99-045 (1999).

No. 39 Kisdi É, Geritz SAH: Evolutionary Branching and Sympatric Speciation in Diploid Populations. IIASA Interim Report IR-99-048 (1999).

No. 40 Ylikarjula J, Heino M, Dieckmann U: Ecology and Adaptation of Stunted Growth in Fish. IIASA Interim Report IR-99-050 (1999). Evolutionary Ecology 13:433-453 (1999).

No. 41 Nowak MA, Sigmund K: Games on Grids. IIASA Interim Report IR-99-038 (1999). Dieckmann U, Law R, Metz JAJ (eds): The Geometry of Ecological Interactions: Simplifying Spatial Complexity, Cambridge University Press, Cambridge, UK, pp. 135-150 (2000).

No. 42 Ferrière R, Michod RE: Wave Patterns in Spatial Games and the Evolution of Cooperation. IIASA Interim Report IR-99-041 (1999). Dieckmann U, Law R, Metz JAJ (eds): The Geometry of Ecological Interactions: Simplifying Spatial Complexity, Cambridge University Press, Cambridge, UK, pp. 318-332 (2000).

No. 43 Kisdi É, Jacobs FJA, Geritz SAH: Red Queen Evolution by Cycles of Evolutionary Branching and Extinction. IIASA Interim Report IR-00-030 (2000). Selection 2:161176 (2001).

No. 44 Meszéna G, Kisdi É, Dieckmann U, Geritz SAH, Metz JAJ: Evolutionary Optimisation Models and Matrix Games in the Unified Perspective of Adaptive Dynamics. IIASA Interim Report IR-00-039 (2000). Selection 2:193-210 (2001).

No. 45 Parvinen K, Dieckmann U, Gyllenberg M, Metz JAJ: Evolution of Dispersal in Metapopulations with Local Density Dependence and Demographic Stochasticity. IIASA Interim Report IR-00-035 (2000). Journal of Evolutionary Biology $16: 143-153$ (2003).

No. 46 Doebeli M, Dieckmann U: Evolutionary Branching and Sympatric Speciation Caused by Different Types of Ecological Interactions. IIASA Interim Report IR-00-040 (2000). The American Naturalist 156:S77-S101 (2000).

No. 47 Heino M, Hanski I: Evolution of Migration Rate in a Spatially Realistic Metapopulation Model. IIASA Interim Report IR-00-044 (2000). The American Naturalist 157:495511 (2001).

No. 48 Gyllenberg M, Parvinen K, Dieckmann U: Evolutionary Suicide and Evolution of Dispersal in Structured Metapopulations. IIASA Interim Report IR-00-056 (2000). Journal of Mathematical Biology 45:79-105 (2002).

No. 49 van Dooren TJM: The Evolutionary Dynamics of Direct Phenotypic Overdominance: Emergence Possible, Loss Probable. IIASA Interim Report IR-00-048 (2000). Evolution 54: 1899-1914 (2000).

No. 50 Nowak MA, Page KM, Sigmund K: Fairness Versus Reason in the Ultimatum Game. IIASA Interim Report IR00-57 (2000). Science 289:1773-1775 (2000).

No. 51 de Feo O, Ferrière R: Bifurcation Analysis of Population Invasion: On-Off Intermittency and Basin Riddling. IIASA Interim Report IR-00-074 (2000). International Journal of Bifurcation and Chaos 10:443-452 (2000). 
No. 52 Heino M, Laaka-Lindberg S: Clonal Dynamics and Evolution of Dormancy in the Leafy Hepatic Lophozia Silvicola. IIASA Interim Report IR-01-018 (2001). Oikos 94:525-532 (2001).

No. 53 Sigmund K, Hauert C, Nowak MA: Reward and Punishment in Minigames. IIASA Interim Report IR-01-031 (2001). Proceedings of the National Academy of Sciences of the USA 98:10757-10762(2001).

No. 54 Hauert C, De Monte S, Sigmund K, Hofbauer J: Oscillations in Optional Public Good Games. IIASA Interim Report IR-01-036 (2001).

No. 55 Ferrière R, Le Galliard J: Invasion Fitness and Adaptive Dynamics in Spatial Population Models. IIASA Interim Report IR-01-043 (2001). Clobert J, Dhondt A, Danchin E, Nichols J (eds): Dispersal, Oxford University Press, pp. 57-79 (2001).

No. 56 de Mazancourt C, Loreau M, Dieckmann U: Can the Evolution of Plant Defense Lead to Plant-Herbivore Mutualism. IIASA Interim Report IR-01-053 (2001). The American Naturalist 158: 109-123 (2001).

No. 57 Claessen D, Dieckmann U: Ontogenetic Niche Shifts and Evolutionary Branching in Size-Structured Populations. IIASA Interim Report IR-01-056 (2001). Evolutionary Ecology Research 4:189-217 (2002).

No. 58 Brandt H: Correlation Analysis of Fitness Landscapes. IIASA Interim Report IR-01-058 (2001).

No. 59 Dieckmann U: Adaptive Dynamics of Pathogen-Host Interacations. IIASA Interim Report IR-02-007 (2002). Dieckmann U, Metz JAJ, Sabelis MW, Sigmund K (eds): Adaptive Dynamics of Infectious Diseases: In Pursuit of Virulence Management, Cambridge University Press, Cambridge, UK, pp. 39-59 (2002).

No. 60 Nowak MA, Sigmund K: Super- and Coinfection: The Two Extremes. IIASA Interim Report IR-02-008 (2002). Dieckmann U, Metz JAJ, Sabelis MW, Sigmund K (eds): Adaptive Dynamics of Infectious Diseases: In Pursuit of Virulence Management, Cambridge University Press, Cambridge, UK, pp. 124-137 (2002).

No. 61 Sabelis MW, Metz JAJ: Perspectives for Virulence Management: Relating Theory to Experiment. IIASA Interim Report IR-02-009 (2002). Dieckmann U, Metz JAJ, Sabelis MW, Sigmund K (eds): Adaptive Dynamics of Infectious Diseases: In Pursuit of Virulence Management, Cambridge University Press, Cambridge, UK, pp. 379-398 (2002).

No. 62 Cheptou P, Dieckmann U: The Evolution of SelfFertilization in Density-Regulated Populations . IIASA Interim Report IR-02-024 (2002). Proceedings of the Royal Society of London Series B 269:1177-1186 (2002).

No. 63 Bürger R: Additive Genetic Variation Under Intraspecific Competition and Stabilizing Selection: A Two-Locus Study. IIASA Interim Report IR-02-013 (2002). Theoretical Population Biology 61:197-213 (2002).

No. 64 Hauert C, De Monte S, Hofbauer J, Sigmund K: Volunteering as Red Queen Mechanism for Co-operation in Public Goods Games. IIASA Interim Report IR-02-041 (2002). Science 296:1129-1132(2002).

No. 65 Dercole F, Ferrière R, Rinaldi S: Ecological Bistability and Evolutionary Reversals under Asymmetrical Competition. IIASA Interim Report IR-02-053 (2002). Evolution 56:1081-1090 (2002).
No. 66 Dercole F, Rinaldi S: Evolution of Cannibalistic Traits: Scenarios Derived from Adaptive Dynamics. IIASA Interim Report IR-02-054 (2002). Theoretical Population Biology 62:365-374 (2002).

No. 67 Bürger R, Gimelfarb A: Fluctuating Environments and the Role of Mutation in Maintaining Quantitative Genetic Variation. IIASA Interim Report IR-02-058 (2002). Genetical Research 80:31-46 (2002).

No. 68 Bürger R: On a Genetic Model of Intraspecific Competition and Stabilizing Selection. IIASA Interim Report IR02-062 (2002). Amer. Natur. 160:661-682 (2002).

No. 69 Doebeli M, Dieckmann U: Speciation Along Environmental Gradients. IIASA Interim Report IR-02-079 (2002). Nature 421:259-264 (2003).

No. 70 Dercole F, Irisson J, Rinaldi S: Bifurcation Analysis of a Prey-Predator Coevolution Model. IIASA Interim Report IR-02-078 (2002). SIAM Journal on Applied Mathematics 63:1378-1391 (2003).

No. 71 Le Galliard J, Ferrière R, Dieckmann U: The Adaptive Dynamics of Altruism in Spatially Heterogeneous Populations. IIASA Interim Report IR-03-006 (2003). Evolution 57:1-17 (2003).

No. 72 Taborsky B, Dieckmann U, Heino M: Unexpected Discontinuities in Life-History Evolution under SizeDependent Mortality. IIASA Interim Report IR-03-004 (2003). Proceedings of the Royal Society of London Series B 270:713-721 (2003).

No. 73 Gardmark A, Dieckmann U, Lundberg P: LifeHistory Evolution in Harvested Populations: The Role of Natural Predation. IIASA Interim Report IR-03-008 (2003). Evolutionary Ecology Research 5:239-257 (2003).

No. 74 Mizera F, Meszéna G: Spatial Niche Packing, Character Displacement and Adaptive Speciation Along an Environmental Gradient. IIASA Interim Report IR-03-062 (2003). Evolutionary Ecology Research 5: 363-382 (2003).

No. 75 Dercole F: Remarks on Branching-Extinction Evolutionary Cycles. IIASA Interim Report IR-03-075 (2003). Journal of Mathematical Biology 47: 569-580 (2003).

No. 76 Hofbauer J, Sigmund K: Evolutionary Game Dynamics. IIASA Interim Report IR-03-078 (2003). Bulletin of the American Mathematical Society 40: 479-519 (2003).

No. 77 Ernande B, Dieckmann U, Heino M: Adaptive Changes in Harvested Populations: Plasticity and Evolution of Age and Size at Maturation. IIASA Interim Report IR03-058 (2003). Proceedings of the Royal Society of London Series B-Biological Sciences, 271: 415-423 (2004).

No. 78 Hanski I, Heino M: Metapopulation-Level Adaptation of Insect Host Plant Preference and Extinction-Colonization Dynamics in Heterogeneous Landscapes. IIASA Interim Report IR-03-028 (2003). Theoretical Population Biology 63:309-338 (2003).

No. 79 van Doorn G, Dieckmann U, Weissing FJ: Sympatric Speciation by Sexual Selection: A Critical Re-Evaluation. IIASA Interim Report IR-04-003 (2004). American Naturalist 163: 709-725 (2004).

No. 80 Egas M, Dieckmann U, Sabelis MW: Evolution Restricts the Coexistence of Specialists and Generalists - the Role of Trade-off Structure. IIASA Interim Report IR-04-004 (2004). American Naturalist 163: 518-531 (2004). 
No. 81 Ernande B, Dieckmann U: The Evolution of Phenotypic Plasticity in Spatially Structured Environments: Implications of Intraspecific Competition, Plasticity Costs, and Environmental Characteristics. IIASA Interim Report IR-04-006 (2004). Journal of Evolutionary Biology 17 (3): 613-628 (2004).

No. 82 Cressman R, Hofbauer J: Measure Dynamics on a One-Dimensional Continuous Trait Space: Theoretical Foundations for Adaptive Dynamics. IIASA Interim Report IR04-016 (2004).

No. 83 Cressman R: Dynamic Stability of the Replicator Equation with Continuous Strategy Space. IIASA Interim Report IR-04-017 (2004).

No. 84 Ravigné V, Olivieri I, Dieckmann U: Implications of Habitat Choice for Protected Polymorphisms. IIASA Interim Report IR-04-005 (2004). Evolutionary Ecology Research 6: 125-145 (2004).
No. 85 Nowak MA, Sigmund K: Evolutionary Dynamics of Biological Games. IIASA Interim Report IR-04-013 (2004). Science 303: 793-799 (2004).

No. 86 Vukics A, Asbóth J, Meszéna G: Speciation in Multidimensional Evolutionary Space. IIASA Interim Report IR-04-028 (2004). Physical Review E 684 (2003).

No. 87 de Mazancourt C, Dieckmann U: Trade-off Geometries and Frequency-dependent Selection. IIASA Interim Report IR-04-039 (2004).

No. 88 Cadet CR, Metz JAJ, Klinkhamer PGL: Size and the Not-So-Single Sex: disentangling the effects of size on sex allocation. IIASA Interim Report IR-04-084 (2004). American Naturalist, 164: 779-792 (2004).

No. 89 Rueffler C, van Dooren TJM, Metz JAJ: Adaptive Walks on Changing Landscapes: Levins' Approach Extended. IIASA Interim Report IR-04-083 (2004). Theoretical Population Biology, 65: 165-178 (2004).

Issues of the IIASA Studies in Adaptive Dynamics series can be obtained at www.iiasa.ac.at/Research/ADN/Series.html or by writing to adn@iiasa.ac.at. 


\section{Contents}

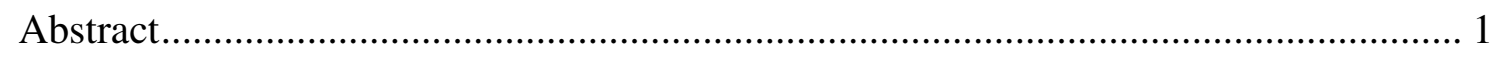

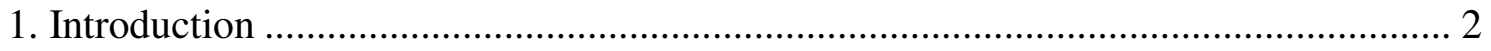

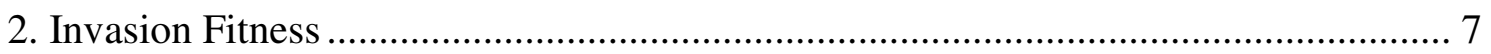

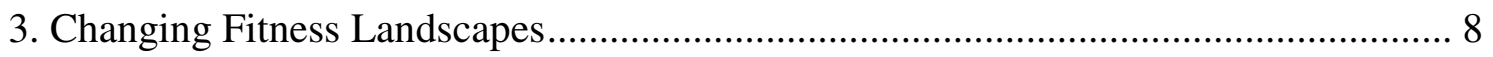

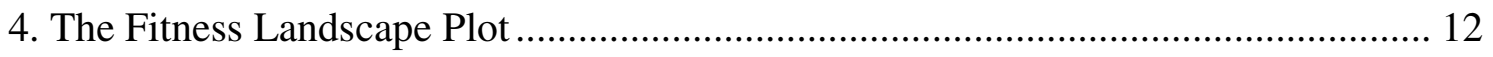

5. Polymorphic Evolution of a One-Dimensional Trait ................................................ 17

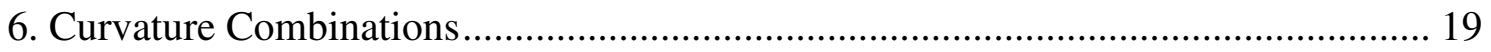

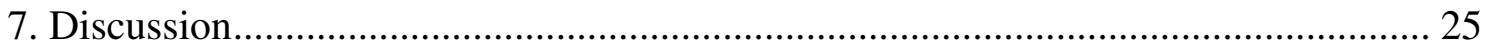

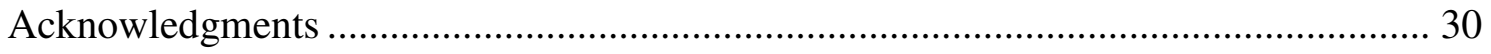

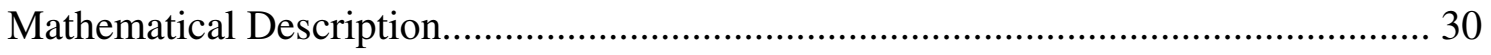

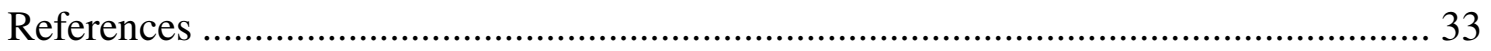




\title{
Adaptive Walks on Changing Landscapes: Levins' Approach Extended
}

\author{
C. Rueffler ${ }^{\mathrm{a}, *}$, T. J. M. Van Dooren ${ }^{\mathrm{b}}$, J. A. J. Metz ${ }^{\mathrm{a}, \mathrm{c}}$ \\ a Section Theoretical Biology, Institute of Biology, Leiden University, Kaiserstraat \\ 63, NL-2311 GP Leiden, The Netherlands \\ ${ }^{\mathrm{b}}$ Section Animal Ecology, Institute of Biology, Leiden University, Kaiserstraat 63, \\ NL-2311 GP Leiden, The Netherlands \\ ${ }^{\mathrm{c}}$ Adaptive Dynamics Network, International Institute of Applied System Analysis, \\ A-2361 Laxenburg, Austria
}

\begin{abstract}
The assumption that trade-offs exist is fundamental in evolutionary theory. Levins (Am. Nat. 96 (1962), 361-373) introduced a widely adopted graphical method for analyzing evolution towards an optimal combination of two quantitative traits, which are traded off. His approach explicitly excluded the possibility of density-dependent and frequency-dependent selection. Here we extend Levins method towards models, which include these selection regimes and where therefore fitness landscapes change with population state. We employ the same kind of curves Levins used: trade-off curves and fitness contours. However, fitness contours are not fixed but a function of the resident traits and we only consider those that divide the trait space into potentially successful mutants and mutants which are not able to invade ('invasion boundaries'). The developed approach allows to make a priori predictions about evolutionary endpoints and about their bifurcations. This is illustrated by applying
\end{abstract}


the approach to several examples from the recent literature.

Key words: adaptive dynamics, fitness landscape, frequency-dependent selection, invasion fitness, life-history evolution, trade-off

\section{Introduction}

The notion of trade-offs is central to evolutionary theory. Without constraints, organisms would evolve to become masters of all traits. As they do not, organisms must have a limited set of possible phenotypes (Maynard Smith, 1978). At the boundary of this set organisms face a trade-off: they can only improve one trait at the expense of the others. There exists a long literature that tells how, given such boundary constraints and given that evolution maximizes some optimization criterion, we can calculate the phenotypes that we should expect to evolve (e.g. Maynard Smith, 1978; Stephens and Krebs, 1986; Lessells, 1991; Roff, 1992; Stearns, 1992; Alexander, 1996; McNamara et al., 2001).

Related to optimality models is the notion of fitness landscape or adaptive landscape, originally introduced in the context of population genetics (Wright, 1931; Lande, 1976). In phenotypic evolution a fitness landscape is the visualization of the function which assigns to every point in trait space the corresponding value of the optimization criterion. If the trait space is two-dimensional, this can indeed be seen as a topographical map. On the assumption that the genetic variation is relatively small, traits in such a landscape move uphill in the course of evolution. Evolution stops when the trait of a population has

\footnotetext{
* Corresponding Author.
}

Email address: rueffler@rulsfb.leidenuniv.nl (C. Rueffler). 
either reached a hilltop or a maximum at the boundary of the, constrained, trait space (for an example where such a landscape is calculated see Benkman (1993)).

Levins $(1962 ; 1968)$ introduced an influential graphical method for finding the optimal strategy if reproduction takes place in two different environments (Fig. 1). The two dimensions of his trait space are the average number of offspring a given phenotype produces in each type of environment. Levins called the set of feasible combinations 'fitness set'. Two ingredients are important in his approach. The first one is the trade-off curve which describes the relation between the reproductive abilities in the two environments. It constitutes a boundary of the trait space. The second one are the contours of the fitness landscape which consist of all points in trait space that yield equal fitness. When these two types of curves are plotted on top of each other the optimal phenotype can be read from this plot. It is the strategy in the fitness set that lies on the highest contour.

This approach appears particularly successful in life-history theory, where it has been applied to the evolution of reproductive effort and the evolution of iteroparity versus semelparity (e.g Pianka and Parker, 1975; Bell, 1980; Takada, 1995) and has found its way into textbooks (Yodzis, 1989, p. 324; Roff, 1992, p.71; 2002, p.197). If fitness contours are linear the general result is as follows: for concave trade-offs an intermediate strategy will be optimal (Fig. 1a), while for convex trade-offs one of the two boundary strategies will be optimal (Fig. 1b).

In life history theory it is traditionally assumed that neither density-dependence nor frequency-dependence influences the process of selection, leading to the 
intrinsic rate of increase $r$ as the natural optimization criterion (Stearns, 1992; Roff, 1992; Charlesworth, 1994). Studies including density-dependence mostly used either the expected lifetime reproductive success $R_{0}$ or population size at equilibrium $\hat{N}$ as the optimization criteria (e.g. Michod, 1979; Charnov, 1993; Charlesworth, 1994). A large part of life history theory has been developed in this way (Roff, 1992, 2002; Stearns, 1992) and has also been corroborated in many aspects by empirical findings (Stearns, 2000).

However, there are two reasons why the optimization approach is not generally applicable. First, there are density-dependent scenarios where no optimization criterion mentioned so far works properly and where other functions must be maximized (Mylius and Diekmann, 1995; Metz et al., 1996b). If the proper criterion is not known, optimization tends to fail. Second, optimization is generally impossible in the presence of frequency-dependence (Fisher, 1930). The study of frequency-dependent evolution made its first big step in behavioral ecology with the introduction of game theory and the ESS-concept (Hamilton, 1967; Maynard Smith and Price, 1973; Maynard Smith, 1982; for a review see Vincent and Brown, 1988). There is now also a growing body of literature emphasizing the potential importance of frequency-dependent selection outside the realm of individual behavior (Kawecki, 1993; Day and Taylor, 1996; Svensson and Sheldon, 1998). Frequency-dependent selection can have as a consequence that evolution leads towards fitness minima (Christiansen, 1991; Abrams et al., 1993) or that fitness maxima are not approached (Hofbauer and Sigmund, 1990; Nowak, 1990). These aspects emerge in a natural way if evolutionary trait substitution sequences are derived from population dynamics. This is done in the theory of adaptive dynamics (Metz et al., 1992, 1996a; Dieckmann and Law, 1996; Geritz et al., 1997, 1998), which may be defined 
as the study of mutation limited trait substitution processes through repeated invasions of mutants in fitness landscapes that keep changing as a result of those very substitutions. The correctness of any optimization criterion has to be proven with respect to the population ecology where it is applied.

The aim of this paper is to extend Levins' graphical approach for the classification of optimal strategies in a systematic way to models which explicitly account for any type of population regulation and therefore any type of density-dependence and frequency-dependence. This classification allows for a priori predictions of the evolutionary end points based on the same types of curves Levins used: trade-off curves and fitness contours. However, fitness contours now do not represent strategies with the same value of an a priori chosen optimization criterion but strategies sharing the same invasion fitness for a given resident population. Invasion fitness is the growth rate of an initially rare mutant in a resident population which is at its ecological equilibrium (Fisher, 1930; Reed and Stenseth, 1984; Metz et al., 1992). In the presence of frequency-dependence the fitness landscape is not fixed but depends on the resident types. We not only determine whether a pure or an intermediate strategy is the optimal one but also classify the dynamics of the evolutionary process, i.e., whether such a strategy is approached. Special attention will be given to the scenario of evolutionary branching where an originally monomorphic population turns dimorphic because of disruptive selection generated by frequency-dependent interactions.

In the study of evolution invasion and Levins' fitness set approach have been brought together in earlier work. However, this has never been developed systematically and in a fully dynamical sense. Lawlor and Maynard Smith (1976) employed the combination in a study on coevolution of competing species. 
Charnov used fitness sets extensively when he developed his theory of sex allocation (Charnov et al., 1976; Charnov, 1982). Sex allocation theory is maybe the field where the signature of frequency-dependence was recognized first (Fisher, 1930). The fitness landscape that a mutant type with a certain sex ratio experiences, necessarily depends on the sex ratio of the resident type. Gatto (1993) and Ferrière and Gatto (1995) linked the two concepts when they investigated which type of population dynamics one can expect to evolve in a simple Ricker model. Another specific example of the approach we develop systematically in this paper can be found in the appendix of de Mazancourt et al. (2001).

The outline of the paper is as follows. In Section 2 we review the concept of invasion fitness and in Section 3 we show how the application of this fitness concept makes fitness landscapes change. After that we develop a complete classification of the possible dynamical behavior of evolution as a function of the curvature of the trade-offs and the curvature of the changing fitness contours. Monomorphic populations are treated in Section 4. In Section 5 we extend the results to polymorphic populations. In Section 6 we demonstrate how the developed approach can be applied. We discuss which evolutionary predictions can be derived a priori for qualitatively different curvature combinations of trade-offs and fitness contours and how changes in curvature can alter evolutionary outcomes. We apply these ideas to several models from the recent literature. 


\section{Invasion Fitness}

The evolutionary success of a mutant depends on whether or not this initially rare type can spread in a given resident population. Evolutionary success is measured in terms of invasion fitness $s\left(\theta_{m}, E_{\theta_{r}}\right)$, i.e., the long term average growth per capita and per time unit of an initially rare mutant with trait vector $\theta_{m}$ in an environment $E_{\theta_{r}}$ shaped by the resident type $\theta_{r}$ (Metz et al., 1992). If the resident population consists of more than one type we call it a coalition $\Theta_{r}=\left(\theta_{1}, \ldots, \theta_{n}\right)$ (Brown and Vincent, 1987a,b). Invasion fitness of a mutant in a resident coalition is then given by $s\left(\theta_{m}, E_{\Theta_{r}}\right)$. The condition of the environment $E_{\Theta_{r}}$ is thereby considered as a function of a population dynamical attractor of the resident types, here assumed to be unique. Note that we do not restrict ourselves to point attractors. It is this attractor that shapes the environment a mutant experiences. For this to hold, we have to make two assumptions (Metz et al., 1992, 1996a). First, there are no long lasting-trends in those environmental components that are not influenced by the resident population. Second, the resident population dynamics has reached its attractor before a new mutant type arrives, i.e., the ecological and evolutionary timescales are separated. Under these conditions we can, with a slight abuse of notation, write $s$ directly as a function of the trait values of the mutant and of the resident population: $s\left(\theta_{m}, \Theta_{r}\right)$. For all types $\theta_{i} \in \Theta_{r}$ of a coalition necessarily

$$
s\left(\theta_{i}, \Theta_{r}\right)=0 .
$$

If $s\left(\theta_{m}, \Theta_{r}\right)>0$ invasion of the mutant is possible and if $s\left(\theta_{m}, \Theta_{r}\right)<0$ the mutant will disappear. If $s\left(\theta_{m}, \Theta_{r}\right)=0$, the mutant is initially neutral with respect to the resident population. Note that our formulation holds for contin- 
uous time measures of fitness. In a discrete time framework, fitness is measured per time step and 0 has to be replaced with 1 .

Whenever invasion occurs we assume that the mutant replaces its progenitor. Sufficient conditions for this to hold are that phenotypic effects of mutations are small and that the evolutionary dynamics are not close to a point where the gradient of invasion fitness (see Eq. (3) below) equals zero (Geritz et al., 2002).

\section{Changing Fitness Landscapes}

The next step is to link invasion fitness with fitness landscapes à la Levins. To this end we first briefly review some notions corresponding to changing fitness landscapes.

When we employ invasion fitness there is no fixed fitness value attached to a type any longer, but every mutant type has an invasion fitness conditional on the resident population. Therefore we do not get a single fitness landscape but as many as there are possible resident populations. For every resident population, the landscape is divided into two parts: (1) a region of types which have invasion fitness larger than zero and are therefore able to invade and (2) a region of types which have invasion fitness smaller than zero and are not able to invade. If the trait space is two-dimensional, one can visualize it as a landscape with a region above sea level and a region below sea level. The shoreline in this landscape is made up of all those mutant strategies which are selectively neutral: they would neither grow nor decline in numbers under the conditions set by the resident population. We will refer to this contour line as 
the 'invasion boundary'. It is given implicitly by

$$
s\left(\left(t_{1}, \ldots, t_{n}\right), \Theta_{r}\right)=0
$$

where the $t$ 's are the separate traits making up the $n$-dimensional trait vector $\theta_{m}$. If $n=2 \mathrm{Eq}$. (2) defines a curve and if $n=3$ Eq. (2) defines a twodimensional manifold, etc. Since for all resident types $\theta_{r} \in \Theta_{r}$ in a coalition Eq. (2) applies, these types all lie on the invasion boundary generated by that coalition.

A successful mutant either replaces one or more types already present in the population, or the number of types coexisting will increase by one. After the coalition has settled on a new population dynamical attractor, Eq. (1) holds for the new set of residents. Each such event corresponds to a step in trait space away from the seashore in an upward direction. However, each time the seashore changes such that all types of the new coalition reside again on the shoreline (Marrow et al., 1992). If one assumes that mutational steps are small, then a sequence of successful invasions gradually changes the fitness landscape.

Hilltops, bottoms of troughs and saddles are points of special interest in a fitness landscape. Resident strategies that sit on one of these special points of the landscape generated by themselves are called 'evolutionarily singular strategies' $\theta_{r}^{*}$ (Metz et al., 1996a; Geritz et al., 1998). These are points where directional selection ceases to act. Mathematically this condition corresponds to a zero fitness gradient,

$$
\left.\frac{\partial s\left(\theta_{m}, \Theta_{r}^{*}\right)}{\partial \theta_{m}}\right|_{\theta_{m}=\theta_{j}^{*}}=0 \quad \text { for all } \theta_{j}^{*} \in \Theta_{r}^{*},
$$

which has the same dimension as the trait vector. If this condition holds 
for all members of a resident community, then this community is called an 'evolutionarily singular coalition' (Metz et al., 1996a; Geritz et al., 1998).

Singular strategies in one-dimensional trait spaces can be classified according to two properties: convergence stability and noninvadability (Metz et al., 1996a; Geritz et al., 1997, 1998). The first property means that a singular strategy is approached in the course of evolution from within a certain neighborhood (Eshel and Motro, 1981; Eshel, 1983; Christiansen, 1991). A singular strategy not convergence stable is repelling. A strategy is (locally) noninvadable if no (nearby) mutant can increase in frequency when the singular strategy is the resident type. This property corresponds to the ESS condition of game theory (Maynard Smith, 1982). Since these two properties are mutually independent (Eshel, 1983; Taylor, 1989; Nowak, 1990; Christiansen, 1991; Abrams et al., 1993) four different types of evolutionarily singular strategies exist:

Continuously stable strategies (CSSs) (Eshel and Motro, 1981; Eshel, 1983; Christiansen, 1991) are both convergence stable and noninvadable. They are end points of the evolutionary dynamics.

Branching Points (Metz et al., 1996a; Geritz et al., 1997, 1998) are convergence stable and invadable. Directional selection drives a population towards such a point. Once it is reached selection becomes disruptive. Any surrounding strategy can invade and coexist with the resident type in a protected polymorphism. The number of resident strategies therefore usually increases by one.

Garden of Eden Points (Hofbauer and Sigmund, 1990; Nowak, 1990) are strategies which are noninvadable but are never approached in the course of evolution. If a Garden of Eden point happens to be the resident strategy, selection is stabilizing. However, any perturbation will lead to directional 
selection away from it.

Repellors are not convergence stable and can be invaded by any surrounding strategy.

In one-dimensional trait spaces a singular point generically corresponds to either a hilltop or to a bottom of a trough. When trait spaces have more dimensions, also saddle points become possible which follow the above classification only in certain directions. Furthermore, not only selection but also the mutational process influences the direction of evolutionary change and the notion of convergence stability becomes more complex (Motro, 1994; Matessi and Di Pasquale, 1996; Leimar, 2001).

Under certain conditions an optimization approach yields the same results as the more general approach of an invasion analysis. The conditions for the existence of a function which gets maximized in the process of evolution were worked out by Mylius and Diekmann (1995) and Metz et al. (1996b). If an optimization criterion exists, two properties hold: (1) The population stays monomorphic. For any set of types, the type with the highest value of the optimization criterion will displace all the others, making a polymorphism impossible. (2) Invasion boundaries coincide with the contour curves of the optimization criterion. An invasion boundary corresponding to a certain resident strategy consists of all strategies which are selectively neutral. These are exactly those strategies which have the same value of the optimization criterion as the given resident strategy. Only in this case the fitness landscape can be visualized as a fixed landmass surrounded by a rising sea, with the resident type always positioned on the shoreline of the sea. 


\section{The Fitness Landscape Plot}

On our way to link Levins' fitness set to the ideas of the previous section, we will develop our graphical approach in detail for two positive traits $\phi$ and $\psi$ and monomorphic populations. From now on we will assume that invasion fitness is an increasing function of both these mutant traits. The feasibility set is limited by the two axes and a trade-off curve. According to the traditional mathematical notation a concave trade-off curve is characterized by a negative second derivative (Fig. 1a). We call such a trade-off weak. It gives rise to a convex feasibility set. A convex or strong trade-off is characterized by a positive second derivative and gives rise to a concave feasibility set (Fig. 1b).

Since fitness is increasing in both traits, the trait vector of a population will in general stay close to the trade-off curve. If an invasion boundary intersects with the trade-off curve then there also exist strategies inside the feasibility set which are able to invade. However, when mutational steps are small, steps in the direction of the trade-off curve will dominate and the traits will stay close to the boundary. We will idealize this with the simplifying assumption that, after approaching it, evolution further proceeds along the trade-off curve. Thus an increase in one trait is only possible at the expense of an immediate decrease in the other one.

The trade-off curve is a one-dimensional manifold with boundary, which we can parameterize with a single variable $\theta \in[0,1]$. Every value of $\theta$ represents a point $(\phi, \psi)$ in trait space lying on the trade-off curve. We parameterize the trade-off curve corresponding to the direction of reading, from the top left to the bottom right. The point $\theta=0$ then corresponds to the minimum of $\phi$ 
and to the maximum of $\psi$ while $\theta=1$ corresponds to the maximum of $\phi$ and the minimum of $\psi$ (Fig. 2). With this convention, strategies corresponding to smaller $\theta$-values than a given strategy lie above and to the left of it. Biologically, $\theta$ might have the interpretation of effort invested in one biological function and thereby not invested in the other function. For later usage we introduce another convention already here. An invasion boundary is said to intersect with the trade-off curve from the inside of the feasibility set if the invasion boundary for low values of $\phi$ lies inside the feasibility set and intersects with the trade-off curve as $\phi$ increases. An invasion boundary is said to intersect from the outside if the opposite holds true.

If an optimization criterion exists, the dynamics of the evolutionary process can be viewed as hill climbing on a fixed landscape. This scenario corresponds to the theory developed by Levins $(1962,1968)$ and we describe it here first. Evolution proceeds along the trade-off curve until a strategy corresponding to a (local) fitness maximum is reached. This evolutionary end point is a CSS. No strategy on a higher contour can be reached. If contours are linear and the trade-off curve concave, the CSS is either an intermediate strategy (Fig. 1a) or one of the boundary strategies. The first holds true when there is a point on the trade-off curve where a contour line is a tangent to the tradeoff curve. If the trade-off curve is convex, either both boundary strategies or only one of them are CSSs (Levins, 1962) (Fig. 1b). Again, the first applies when there exists a $\theta$ where the contour is a tangent of the trade-off curve. This point corresponds to a Repellor. In general, one of the two boundary strategies will have a higher fitness value than the other. However, depending on the starting condition and the occurring mutations, that strategy may not be attainable. When the trade-off curve has concave and convex parts, 
combinations of intermediate and boundary CSSs can occur (Schaffer, 1974). If contour lines are not linear other results are also possible (Levins, 1962).

The more general case is that the fitness landscape depends on the resident strategy. The invasion boundary corresponding to a specific resident strategy determines which mutants are able to invade and which ones are not. Once the trade-off curve is reached, the direction of further trait substitutions depends on the way the invasion boundaries intersect with the trade-off curve (see Fig. $2 \& 3)$. If they intersect from the outside of the feasibility set, mutants with a larger $\theta$ (i.e., with a trait combination on the trade-off curve below the resident strategy) are able to invade. If it intersects from the inside of the feasibility set, mutants with a smaller $\theta$ are able to invade. This process continues until the invasion boundary becomes tangent to the trade-off curve or until the border of the trait space is attained. In the first case an evolutionarily singular strategy is reached. The classification from Section 3 can now be made in terms of the behavior of the invasion boundaries. Whether a singular strategy is noninvadable or not, depends on whether the corresponding fitness contour is locally outside or inside the feasibility set near the point of tangency. In the first case the resident strategy is a fitness maximum and therefore noninvadable, in the second case the strategy is a minimum and invadable. Whether a singular strategy is convergence stable or not, depends on the behavior of the invasion boundaries attached to the surrounding strategies on the tradeoff curve. An evolutionarily singular strategy is convergence stable if invasion boundaries for nearby resident strategies intersect with the trade-off curve in such a way that strategies closer to the evolutionarily singular strategy can invade (Fig. 2a, b \& 3a, b, c, d). This is the case if invasion boundaries corresponding to strategies with a smaller $\theta$ than the singular strategy intersect 
with the trade-off curve from the outside. The opposite has to hold for strategies with a larger $\theta$ than the singular strategy. The reverse pattern means that a strategy is repelling (Fig. 2c, d \& 3e, f, g, h).

Since the above-mentioned properties are mutually independent we can encounter all four different combinations. First we consider the simplest case where invasion boundaries are linear, a condition commonly encountered in models from the literature. Later on we treat the more general case where no restrictions are put on the curvature of the invasion boundaries. If the tradeoff curve is concave, any tangent invasion boundary lies outside the feasibility set, of course with the exception of the point of tangency. Hence, any singular strategy has to be either a CSS (Fig. 2a) or a Garden of Eden (Fig. 2c). Vice versa, if the trade-off curve is convex, any tangent invasion boundary lies inside the feasibility set. A singular strategy therefore has to be either a Branching Point (Fig. 2b) or a Repellor (Fig. 2d).

For curved invasion boundaries any type of singular strategy can occur on concave trade-offs as well as on convex trade-offs. If the curvature of either the trade-off curve or of the invasion boundary changes over trait space, our classification holds only locally.

CSS The invasion boundary is tangent to the trade-off curve from the outside of the feasibility set. Invasion boundaries attached to points above the singular strategy intersect from the outside with the trade-off curve and from the inside below the singular strategy. If the trade-off curve is concave, invasion boundaries have to be less concave (Fig. 3a). If the trade-off curve is convex, invasion boundaries have to be more convex (Fig. 3b).

Branching point The invasion boundary is tangent to the trade-off curve 
from the inside of the feasibility set. Invasion boundaries attached to points above the singular strategy intersect from the outside with the trade-off curve and vice versa below the singular strategy. If the trade-off curve is concave, invasion boundaries have to be more concave (Fig. 3c). If the tradeoff curve is convex, invasion boundaries have to be less convex (Fig. 3d).

Garden of Eden Points The invasion boundary is tangent to the trade-off curve from the outside of the feasibility set. Invasion boundaries attached to points above the strategy intersect from the inside with the trade-off curve and from the outside below the strategy. If the trade-off curve is concave, invasion boundaries have to be less concave (Fig. 3e). If the trade-off curve is convex, invasion boundaries have to be more convex (Fig. 3f).

Repellor The invasion boundary is tangent to the trade-off curve from the inside of the feasibility set. Invasion boundaries attached to points above the singular strategy intersect from the inside with the trade-off curve and from the outside below the singular strategy. If the trade-off curve is concave, invasion boundaries have to be more concave (Fig. 3g). If the trade-off curve is convex, invasion boundaries have to be less convex (Fig. 3h).

The different properties of strategies that lie on the trade-off curve can also be expressed in terms of the derivatives of the invasion boundaries and the trade-off curve at the points where they intersect. For a strategy being a singular point the first derivative of the two curves have to be equal. Whether or not such a strategy is invadable depends on the relative curvature, i.e., the difference in curvature between the two curves. This can be determined by means of the second derivatives, which is worked out in detail in the appendix.

We now have a full classification of evolutionary singular points with respect to the curvature of the trade-off curve and the shape of the invasion boundaries 
of the fitness landscape. On the assumption that standing genetic variation is negligible, such a graphical classification is usually done in terms of socalled pairwise invasibility plots (PIPs) (Metz et al., 1996a; Dieckmann, 1997; Geritz et al., 1997, 1998). Introduced by Christiansen (1980), Motro (1982), Matsuda (1985) and van Tienderen and de Jong (1986), they now are used widely to visualize evolutionary dynamics (e.g. Claessen and Dieckmann, 2002; Day et al., 2002; Egas et al., in press). Their strength is that they allow for an easy graphical analysis of evolutionary dynamics in one-dimensional trait spaces and for monomorphic populations. However, the predictions of evolutionary dynamics which are possible with the fitness landscape plot (see Section 6) cannot be extracted from PIPs. While a PIP is simply a contour plot of invasion fitness, the fitness landscape plot allows us to derive evolutionary dynamics from more mechanistic arguments. Since it is based on the shape of the trade-off curve and the invasion boundaries, we can assess how the evolutionary dynamics changes when their curvatures are altered. The PIP shows invasion fitness only for resident and mutant strategies lying on the trade-off curve, i.e., for the strategies which we parameterized by $\theta$. In Fig. 4 we illustrate the relation between a fitness landscape plot and a PIP.

\section{Polymorphic Evolution of a One-Dimensional Trait}

Once a population has reached a Branching Point, selection becomes disruptive and a formerly monomorphic population turns dimorphic (Metz et al., 1996a; Geritz et al., 1998). From then on, we have to follow the evolution of two resident types $\theta_{1}$ and $\theta_{2}$. Because of Eq. (1), both resident types must lie on the same invasion boundary. Further evolutionary dynamics can be read from 
a fitness landscape plot in the same way as was done from Fig. 2 and Fig. 3. The population has reached an evolutionarily singular coalition $\left(\theta_{1}^{*}, \theta_{2}^{*}\right)$ when

$$
\left.\frac{\partial s\left(\theta_{m},\left(\theta_{1}^{*}, \theta_{2}^{*}\right)\right)}{\partial \theta_{m}}\right|_{\theta_{m}=\theta_{j}^{*}}=0 \quad \text { for } j \in\{1,2\} .
$$

At an evolutionarily singular coalition, the invasion boundary has to be tangent to the trade-off curve at both resident strategies. Further branching can occur if the invasion boundary is tangent to the trade-off curve from the inside of the feasibility set for one of the two resident types at least. Eq. (4) immediately generalizes to evolutionarily singular coalitions with an arbitrary number of resident types.

Under certain conditions the course of the polymorphic evolution can be derived from geometrical arguments, for instance when the invasion boundaries for populations consisting of two resident types are linear. Such an invasion boundary always intersects with the trade-off curve in the two points corresponding to the two resident types. If the trade-off curve is convex, the traits in the singular coalition are either driven apart until the boundary of the trait space is reached (Fig. 5) or until one of the two types goes extinct. In the first case the end point of the evolutionary process corresponds to a population consisting of the two pure strategies $\theta_{1}^{*}=0$ and $\theta_{2}^{*}=1$. No further branching is possible. The second case is sketched in Fig. 6, on the assumption that the population decline is continuous. At the point where the evolutionary trajectory changes from dimorphic to monomorphic by the extinction of one type, the invasion boundary intersects with the trade-off curve in such a way, that selection favors further specialization of the remaining type (Fig. 6) until it has reached the boundary of the trait space. Let us further assume that the dimorphism emerged at a Branching Point, which by definition at- 
tracts monomorphic populations from both directions. Under this condition, we can conclude that the gradual extinction of one type is only possible when a Repellor exists between the remaining type and the Branching Point. This Repellor separates the two basins of attraction of the monomorphic evolution, which lead to either the Branching Point or the boundary of the trait space. A second conclusion arising from this argument is, that evolutionary cycles of branching and extinction as observed by Mathias and Kisdi (1999), Kisdi et al. (2000) and Doebeli and Dieckmann (2000) are not possible.

These arguments hold also for nonlinear invasion boundaries, whenever the invasion boundary of the dimorphic population intersects with the trade-off curve from the inside at the upper left resident strategy and from the outside at the lower right resident strategy. A change of such a constellation is only possible via a phase with three points of intersection between the trade-off curve and the invasion boundary. It is only under this condition that the presented results may depend on the rate of evolution in the two types.

It is easy to understand that an evolutionarily singular coalition where two resident types are intermediate strategies requires more complicated curvatures than we discussed so far.

\section{Curvature Combinations}

Our approach elucidates the interplay between population dynamics (invasion fitness) and properties of the organism (trade-off curve) in a graphical way. In certain cases the qualitative outcome of an evolutionary model can be predicted immediately. This is sometimes possible even for polymorphic pop- 
ulations where up to now numerical calculations needed to be employed. In other cases it enables us to predict changes in the outcome of an evolutionary model when parameters which influence the curvature of either the trade-off or the invasion boundaries are altered. That means, it allows us to make statements which are similar to those derived from a bifurcation analysis. In this section we classify models with respect to the curvature of the trade-off and the invasion boundaries into four groups and discuss which results one can derive a priori.

\section{Linear Trade-Off \& Linear Invasion Boundaries}

This is the simplest case. A trade-off curve is linear when one trait is a linear function of the other. An example for this are seeds that can either germinate or stay in the seed bank. The probabilities for these two alternatives have to add up to one. Invasion boundaries are linear when invasion fitness is a linear function of the two traits that are traded off. Sometimes this is easier to detect when a sign-equivalent proxy for invasion fitness is used. In this scenario, an invasion boundary either intersects with the trade-off curve or coincides with it. The first situation corresponds to directional selection while the latter situation corresponds to a singular strategy where all mutants on the trade-off curve are selectively neutral. This singular strategy is a mixed strategy and its value determines the probability of each individual to exhibit either of the pure strategies. It can be repelling or attracting. If such a strategy exists, the corresponding PIP shows a vertical zero contour line next to the diagonal where

the mutant and resident have equal trait values (Geritz et al., 1998; Meszéna et al., 2001). In this case the fitness landscape changes depending on the resident strategy and therefore frequency-dependent selection has to be involved. However, evolutionary branching and genetically polymorphic populations are 
not possible. If no frequency-dependence is present, the evolutionary problem can be solved by maximizing an optimization criterion (Metz et al., 1996b) and either of the two pure strategies will be optimal. The model in Heino et al. (1997) serves as an example for these statements. These authors investigate the evolutionary dynamics of maturation in a semelparous organism. Each organism matures with a certain probability and then dies, or it delays maturation to the next season where it reproduces and then dies. It is assumed that the population is in population dynamical equilibrium so that we can use life-time offspring production $R_{0}$ as a stand-in for invasion fitness. $R_{0}$ equals the sum of the reproductive output at ages one and two, weighted with their respective probabilities. As these probabilities sum up to one, Heino et al.'s model falls into the case where the feasibility set is given by a linear trade-off. When only one resource is involved in the feedback environment there is no frequency-dependence and generically either of the two pure strategies is a CSS, resulting in an annual semelparous organism or a biennial semelparous organism. When two resources are involved a resident strategy can exist for which the slopes of the trade-off and the invasion boundary are equal. This is an evolutionarily singular strategy which is convergence stable, and once it is reached all mutants are selectively neutral. In this situation a certain fraction of the population reproduces after one year while the remaining fraction reproduces after two years.

\section{Nonlinear Trade-Off \& Linear Invasion Boundaries}

Many classical life-history models (e.g Pianka and Parker, 1975; Bell, 1980; Takada, 1995) belong to the category where the trade-off can be either convex or concave and the invasion boundaries are linear. Only CSSs or Gardens of Eden are possible in combination with concave trade-offs (Fig. 2a, c) while 
Branching Points and Repellors are possible in combination with convex tradeoffs (Fig. 2b, d). Whenever the trade-off switches from concave to convex an uninvadable strategy loses this property and becomes either a Branching Point or a Repellor. If frequency-dependence can be excluded we are back at the classical result (Fig. 1). A recent model with a nonlinear trade-off and linear invasion boundaries is presented in Day et al. (2002). These authors developed a model for a simple food chain in order to study the evolutionary implications of different complexities in the interactions between the three different trophic levels. The evolving trait determines the trade-off between growth and reproduction in the consumer. In their model invasion boundaries are linear. This can be seen when calculations are done in terms of the lifetime number of offspring, which in their model is a sign-equivalent proxy for invasion fitness. For convex trade-off curves they either find a Repellor (Fig. 1b and 2d) or a Branching Point (Fig. 2b), depending on whether the interactions in the food chain allow for frequency-dependent selection or not. For concave trade-off curves they always find CSSs (Fig. 1a and Fig. 2a). Our type of analysis (Fig. 5) makes clear that after branching the dimorphic evolution always proceeds until a coalition of the two extreme types is reached where one type invests all energy into reproduction and the other type invests all energy into growth. Day et al. (2002) already surmised this result on the basis of numerical calculations. Our approach not only provides a proof, but also makes it easy to spot the result from the fact that the trade-off is convex and that invasion boundaries stay linear for dimorphic populations.

\section{Linear Trade-Off \& Nonlinear Invasion Boundaries}

Nonlinear invasion boundaries can occur if invasion fitness is a nonlinear function of the two traits that are traded off. In some cases invasion boundaries 
may become linear after rescaling the traits. An example where such a rescaling is not possible, can be found in Ebenman et al. (1996). These authors investigated a model for the evolution of different sensitivities to density dependence using nonlinear Ricker type functions. Nonlinear invasion boundaries also occur in models incorporating environmental fluctuations (Mathias and Kisdi, 2002) or nonequilibrium population dynamics (Gatto, 1993). Ferrière and Gatto (1995) present a theorem which states necessary conditions for invasion boundaries to be convex in models dealing with either fluctuating environments or nonequilibrium dynamics. This is the case when all positive entries of the population matrices are log-concave functions of the evolving traits. If the conditions of the theorem are fulfilled and the trade-off is either linear or concave, Branching Points and Repellors cannot occur.

When nonlinear invasion boundaries occur together with a linear trade-off, again only two of the four types of singular points are possible for each shape of the invasion boundaries. An uninvadable strategy loses the ESS-property whenever the invasion boundary attached to it switches from convex to concave. Therefore a CSS and a Garden of Eden turn into either a Branching Point or Repellor (see Fig. 3, however with linear trade-off). Mathias and Kisdi (2002) analyzed a model with a linear trade-off and nonlinear invasion boundaries. It is a two-patch model with environmental stochasticity for the germination rate of plant seeds, where no analytical expression for the invasion boundaries can be derived. The trade-off determines whether a seed germinates or stays in the seed bank. Since each seed follows either of these two strategies, the feasibility set is given by the straight line where the sum of the two probabilities equals one. The authors perform a numerical bifurcation analysis for the dispersal parameter between the two patches. If dispersal is 
low, evolutionary branching occurs. When dispersal increases, the Branching Point turns into a CSS. From this we can conclude that at the bifurcation point invasion boundaries change from concave to convex (see Fig. 3c and d vs $3 \mathrm{a}$ and $\mathrm{b})$.

\section{Nonlinear Trade-Off \& Nonlinear Invasion Boundaries}

The most general case is where both the trade-off curve and the invasion boundaries are nonlinear (see Fig. 3). A general prediction in this case is that invadable singular strategies occur more easily in combination with strong trade-offs since it is then more likely that an invasion boundary is tangent to the trade-off curve from the inside of the feasibility set. Hence, we predict that uninvadable singular points become invadable when the trade-off is strengthened. This prediction is confirmed in a study by Egas et al. (in press). They study the evolution of specialization in a two-patch model, a situation which corresponds to the models Levins used to present his original ideas. The evolving trait is a parameter determining the efficiencies in the two different habitats which are traded off. Due to environmental stochasticity in their model, it is not possible to derive an analytical expression for the invasion boundaries. They find branching as well on strong and weak trade-off curves. We can conclude that in the latter case invasion boundaries are more strongly concave than the trade-off (Fig. 3c). Whenever they find a CSS, it turns into a Branching Point when the strength of the trade-off is increased sufficiently as predicted by our framework. Furthermore they report of regions in their parameter space where evolutionary branching leads to an interior pair of generalists indicating complexly curved invasion boundaries.

When the shape of the invasion boundaries is known to be either convex or 
concave stronger statements can be made. As an example serves a paper by Gatto (1993) who investigated a Ricker map with a concave trade-off between adult survival and fecundity. In that model invasion boundaries are either linear (when the resident strategy corresponds to a stable equilibrium) or convex (when the resident strategy corresponds to a cyclic or chaotic attractor). He shows that whether a CSS strategy corresponds to either a stable equilibrium or to a cyclic or chaotic attractor depends crucially on the precise extent of the feasibility set. In the model it is obvious that a CSS corresponding to a stable equilibrium is unique. Gatto cannot exclude the possibility that multiple CSSs are possible when they correspond to cyclic or chaotic attractors. However, when two or more attracting singular strategies exist they have to be separated by either a Repellor or a Garden of Eden. Since these repelling strategies cannot occur under the given constraint that the trade-off is concave and the invasion boundaries are convex, multiple CSSs are not possible.

\section{Discussion}

In this paper we extended the graphical approach of Levins $(1962,1968)$ for the classification of optimal strategies to models which include any type of density-dependence and thereby give rise to frequency-dependent selection. In the framework Levins considered, stable fitness maxima (CSSs) and unstable fitness minima (Repellors) are the only possible types of singular strategies. However, when selection is frequency-dependent unstable fitness maxima (Branching Points) and stable fitness minima (Gardens of Eden) are also possible. We present necessary and sufficient conditions in terms of simple geometrical properties for the occurrence of all different evolutionarily singular 
strategies. Furthermore we extended the approach to polymorphic populations which consist of more than one type.

The strength of the presented approach lies in the possibility to make qualitative predictions about long term evolutionary dynamics using simple geometrical techniques. This allows furthermore to predict how the properties of evolutionary end points change with changes in parameters.

The conditions are derived from the same two types of curves Levins used. (1) Trade-off curves have the same meaning as in Levins' original approach. They delimit the set of possible phenotypes within trait space. Their shape is given by genetic, ontogenetic, physiological and morphological constraints of the organism under study. Unfortunately there is no study known to us which has revealed the details of this curvature for any life history tradeoff in a specific organism. However, these curvatures are central in life history theory (Roff, 1992; Stearns, 1992) which indicates a major gap between theory and empirical knowledge. For morphological traits there are promising data describing the shape of trade-off curves (Benkman, 1993; Schluter, 1993). (2) Our approach relies only on one special contour line of the fitness landscape: the invasion boundary. In trait space this contour separates those phenotypes which are able to invade a given resident population from those that are not. Invasion boundaries are fundamentally different from the contours Levins used since they are conditional on a resident population. This reflects the fact that under frequency-dependent selection the fitness landscape is changing with population state. Although it should in principle be possible to determine the curvature of an invasion boundaries empirically, we are not aware of any such study. In the theoretical sense their curvature is the result of a specific model formulation, i.e., it is determined by the way the trade-off enters the fitness 
function which in turn depends on how exactly each component of fitness contributes to population growth.

Although we considered the shape of the trade-off curve and the invasion boundaries as given properties, they can in principle change over evolutionary time. When we allow for flexibility in of these curves, the dimension of the evolutionary trait space is increased. When fitness is an increasing function of both traits, directional selection will act such, that trade-offs become weaker, i.e., more concave, since then both traits increase simultaneously in value. As a consequence, strong trade-offs can only persist under evolutionary constraints. Since the curvature of invasion boundaries is not a direct model assumption but rather implicitly defined, no parameter exists which determines their curvature directly. However, these curvatures can change due to selection on other parameters which are part of the implicit function defining the invasion boundary. A specific example is provided by Gatto (1993). In his model, invasion boundaries are linear when the resident type corresponds to a equilibrium attractor and convex when the resident type corresponds to a oscillating and chaotic attractor. Under certain conditions the former type can get invaded by the latter type. After fixation of such a mutant, the curvature of the invasion boundary has changed from linear to convex.

For the evolutionary dynamics it is decisive whether the way population growth is regulated causes frequency-dependent selection. With frequencydependent selection, optimization tends to fail. For frequency-dependent selection to occur at least two different resources which influence different age classes or stage classes have to be involved (Heino et al., 1998). Here the word resource has to be understood in the widest possible sense, i.e., including all 
factors involved in population regulation like for instance predators. In order to find conditions conducive to frequency-dependent selection in natural populations, empirical studies should try to pinpoint two things: (1) what are the densities involved in density-dependent population regulation and (2) which stage or age dependent parameters are affected. Frequency-dependent selection can also arise from nonequilibrium population dynamics. If the population dynamical attractor is cyclic, quasi-cyclic, or chaotic, optimization is often not possible, and, moreover, the number of coexisting species can exceed the number of limiting resources (e.g. Koch, 1974; Gatto, 1993; Huisman and Weissing, 1999). In this case the invasion success of a rare mutant depends on the resident type and the fluctuations it creates. Stochastic environmental fluctuations can lead to effective frequency-dependent selection in a similar way (e.g. Levins, 1979; Kisdi and Meszéna, 1993; McNamara, 1995).

We derived a priori predictions of the evolutionary dynamics based on the shape of the trade-off and the invasion boundaries in the previous section. The evolutionary dynamics are especially interesting when they allow for Branching Points where populations undergo disruptive selection and become dimorphic. At Branching Points, biological diversity is generated (Doebeli and Dieckmann, 2000). When evolving traits are related to the usage of different resources, branching leads to the evolution of specialists that each rely on a different resource. The occurrence of Branching Points is facilitated by strong trade-offs. If the benefit from each resource is negatively frequency-dependent, evolution will first lead towards a generalist strategy. Once the generalist has become established, selections turns disruptive and it pays off to specialize on a single resource. The likelihood of branching decreases if trade-offs are weak. 
In variable environments invasion fitness is given by the dominant Lyapunov exponent (e.g. Metz et al., 1992). In the simplest case of structureless populations this reduces to the geometric mean of the annual growth rates. Since the geometric mean is very sensitive to occasionally low values, evolution favors strategies with a low variance in the annual growth rate. A bet-hedging generalist that relies on more than one resource will in general suffer less from fluctuations than a specialist, which relies on a single resource (Philippi and Seger, 1989; Wilson and Yoshimura, 1994 and references therein). Thus, we expect that fluctuations make evolutionary branching less likely. This is in accordance with a result of Ferrière and Gatto (1995), which states that environmental fluctuations usually generate convex invasion boundaries. In this manner, environmental fluctuations will hamper the scope for branching.

The described patterns change if fitness is not an increasing function in the two traits but decreasing in one or both traits. In that case the trade-off curve does not get approached from the bottom left corner of the feasibility set but from other directions. The results hold when the definition for strong and weak trade-offs is adjusted. For instance, if fitness is a decreasing function of two traits (e.g. mortality and sensitivity to competition) the trade-off curve is approached from the top right corner of the trait space. A strong trade-off now corresponds to a concave trade-off curve. In many cases, however, it will be possible to reparameterize traits in such a way that fitness is again an increasing function of both traits.

If the trait space has more than two dimensions, several trade-offs can be involved. Therefore the boundary of the trait space becomes a higher dimensional manifold and we have to parameterize it in more than one parameter. 
One can still consider a two-dimensional subspace to which the presented results can be applied. Generally however, the evolutionary dynamics in higher dimensional trait spaces become less easy to classify (Motro, 1994; Matessi and Di Pasquale, 1996; Metz et al., 1996a; Leimar, 2001) and it remains open how the presented results can be extended using more elaborate differential geometry. However, the intuitive idea that the relative curvature of the tradeoff manifold and the invasion manifold decide on the direction of selection, should be correct.

\section{Acknowledgements}

CR was supported by the Netherlands Organization of Scientific Research (NWO), grant 810.34.001. TVD and JAJM were supported by the European Training Network ModLife (Modern Life-History Theory and its Application to the Management of Natural Resources), funded through the Human Potential Program of the European Commission (Contract HPRN-CT-2000-00051). Comments from E. van Ast-Gray, T. Day, M. Durinx, M. Egas, R. Ferrière, É. Kisdi, F. Weissing and an anonymous referee improved the manuscript a lot.

\section{A Mathematical Description}

We can produce the classification of Section 4 by characterizing the evolutionary dynamics in terms of the first and second derivative of the trade-off curve and the invasion boundaries. This is done for the case where the resident 
population is monomorphic. We can write the invasion fitness as

$$
s\left(\theta_{m}, \theta_{r}\right)=s\left(\left[\phi\left(\theta_{m}\right), \psi\left(\theta_{m}\right)\right],\left[\phi\left(\theta_{r}\right), \psi\left(\theta_{r}\right)\right]\right),
$$

where $\phi(\theta)$ and $\psi(\theta)$ denote the coordinates in trait space of either the mutant or the resident. In the polymorphic case we have to replace $\theta_{r}$ by $\theta_{i}$ and the conditions have to hold for each $\theta_{i} \in \Theta_{r}$ in order to achieve the same result for singular coalitions.

The derivative of the trade-off curve at strategy $\theta_{r}$ is given by

$$
\mu\left(\theta_{r}\right):=\frac{\partial \psi}{\partial \theta} \frac{\partial \theta}{\partial \phi}\left(\theta_{r}\right)
$$

The invasion boundary corresponding to $\theta_{r}$ consists of all strategies $(\phi, \psi)$ which are selectively neutral and is given by the implicit function theorem applied to Eq. (2). Its slope at $\theta_{r}$ is

$$
\nu\left(\theta_{r}\right):=\left[-\frac{\partial s\left((\phi, \psi), \theta_{r}\right)}{\partial \phi} / \frac{\partial s\left((\phi, \psi), \theta_{r}\right)}{\partial \psi}\right]_{\phi=\phi\left(\theta_{r}\right), \psi=\psi\left(\theta_{r}\right)} .
$$

A resident strategy $\theta_{r}$ is an evolutionary singular strategy $\theta^{*}$ if the corresponding invasion boundary is tangent to the trade-off curve at this point, i.e.

$$
\mu\left(\theta_{r}\right)=\nu\left(\theta_{r}\right)
$$

An evolutionarily singular strategy $\theta^{*}$ is noninvadable if the invasion boundary is tangent with the trade-off curve from the outside of the feasibility set. This is determined by the curvature of the trade-off curve and the invasion boundary. The curvature of a function at a point $\theta$ is given by the second derivative in the tangential direction. However, at a singular point the trade-off curve and the invasion boundary have the same tangent and we can simply compare the 
second derivatives. A straight line then has zero curvature while a concave curve has negative curvature and a convex curve positive curvature. Thus, $\theta^{*}$ is noninvadable if the curvature of the trade-off curve is smaller than the curvature of the invasion boundary:

$$
\frac{\partial(\mu-\nu)}{\partial \phi}\left(\theta^{*}\right)<0
$$

An evolutionarily singular strategy $\theta^{*}$ is convergence stable if locally the corresponding invasion boundaries intersect with the trade-off curve from the outside for $\theta_{r}<\theta^{*}$ and from the inside for $\theta_{r}>\theta^{*}$ (see Fig. $2 \& 3$ ). This implies that the difference between the derivative of the trade-off curve and the derivative of the invasion boundary (i.e. $\mu-\nu$ ) is positive for $\theta_{r}<\theta^{*}$ and negative for $\theta_{r}>\theta^{*}$. This is equivalent to $(\mu-\nu)\left(\theta^{*}\right)=0$ and

$$
\frac{\partial(\mu-\nu)}{\partial \theta_{r}}\left(\theta^{*}\right)<0
$$

The characterization of the evolutionary dynamics by these properties is given in Table 1.

If invasion boundaries are linear the classification from Table 1 becomes simpler. Since the curvature of a straight line is zero the difference in curvature between the trade-off curve and the invasion boundaries is solely determined by the trade-off curve. The condition for noninvadability from Eq. (A5) becomes

$$
\frac{\partial \mu}{\partial \phi}\left(\theta^{*}\right)<0
$$

The curvature of the trade-off curve has to be negative and therefore concave and a singular strategy is then either a CSS or a Garden of Eden. If the tradeoff curve is convex its curvature is positive and a singular strategy can only be a Repellor or a Branching Point. 


\section{References}

Abrams, P. A., Matsuda, H., Harada, Y., 1993. Evolutionary unstable fitness maxima and stable fitness minima of continuous traits. Evolutionary Ecology $7,465-487$.

Alexander, R. M., 1996. Optima for Animals. Princeton, NJ: Princeton University Press.

Bell, G., 1980. The costs of reproduction and their consequences. The American Naturalist 116, 45-76.

Benkman, C. W., 1993. Adaptation to single resources and the evolution of crossbill (Loxia) diversity. Ecological Monographs 63, 305-325.

Brown, J. S., Vincent, T. L., 1987a. Coevolution as an evolutionary game. Evolution 41, 66-79.

Brown, J. S., Vincent, T. L., 1987b. A theory for the evolutionary game. Theoretical Population Biology 31, 140-166.

Charlesworth, B., 1994. Evolution in Age-Structured Populations, 2nd Edition. No. 13 in Cambridge Studies in Mathematical Biology. Cambridge University Press.

Charnov, E. L., 1982. The Theory of Sex Allocation. Monographs in Population Biology. Princeton University Press, Princeton, New Jersy.

Charnov, E. L., 1993. Life History Invariants: Some Explorations of Symmetry in Evolutionary Ecology. Oxford Series in Ecology and Evolution. Oxford University Press, Oxford, U.K.

Charnov, E. L., Maynard Smith, J., Bull, J. J., 1976. Why be an hermaphrodite? Nature 263, 125-126.

Christiansen, F. B., 1991. On conditions for evolutionary stability for a continuously varying character. The American Naturalist 138, 37-50. 
Christiansen, F. B., Loeschke, V., 1980. Evolution and intraspecific exploitation competition I. One-locus theory for small additve gene effects. Theoretical Population Biology 18, 297-313.

Claessen, D., Dieckmann, U., 2002. Ontogenetic niche shifts and evolutionary branching in size-structured populations. Evolutionary Ecology Research 4, $189-217$.

Day, T., Abrams, P. A., Chase, J. M., 2002. The role of size-specific predation in the evolution and diversification of prey life histories. Evolution 56, 877887.

Day, T., Taylor, P. D., 1996. Evolutionarily stable versus fitness maximizing life histories under frequency-dependent selection. Proceedings of the Royal Society of London, B 263, 333-338.

de Mazancourt, C., Loreau, M., Dieckmann, U., 2001. Can the evolution of plant defense lead to plant-herbivore mutualism? The American Naturalist 158, 109-123.

Dieckmann, U., 1997. Can adaptive dynamics invade? Trends in Ecology and Evolution 12, 128-131.

Dieckmann, U., Law, R., 1996. The dynamical theory of coevolution: A derivation from stochastic ecological processes. Journal of Mathematical Biology $34,579-612$.

Doebeli, M., Dieckmann, U., 2000. Evolutionary branching and sympatric speciation caused by different types of ecological interactions. The American Naturalist 156, S77-S101.

Ebenman, B., Johansson, A., Jonsson, T., 1996. Evolution of stable population dynamics through natural selection. Proceedings of the Royal Society London, B 263, 1145-1151.

Egas, M., Dieckmann, U., Sabelis, M. W. in press. Evolution restricts the 
coexistence of specialists and generalists - the role of trade-off structure. The American Naturalist.

Eshel, I., 1983. Evolutionary and continuous stability. Journal of Theoretical Biology 103, 99-111.

Eshel, I., Motro, U., 1981. Kin selection and strong evolutionary stability of mutual help. Theoretical Population Biology 19, 420-433.

Ferrière, R., Gatto, M., 1995. Lyapunov exponents and the mathematics of invasion in oscillatory of chaotic populations. Theoretical Population Biology 48, 126-171.

Fisher, R. A., 1930. The genetical theory of natural selection. At the Clarendon Press, Oxford.

Gatto, M., 1993. The evolutionary optimality of oscillatory and chaotic dynamics in simple population models. Theoretical Population Biology 43, $310-336$.

Geritz, S. A. H., Gyllenberg, M., Jacobs, F. J. A., Parvinen, K., 2002. Invasion dynamics and attractor inheritance. Journal of Mathematical Biology 44, $548-560$.

Geritz, S. A. H., Kisdi, É., Meszéna, G., Metz, J. A. J., 1998. Evolutionarily singular strategies and the adaptive growth and branching of the evolutionary tree. Evolutionary Ecology 12, 35-57.

Geritz, S. A. H., Metz, J. A. J., Kisdi, É., Meszéna, G., 1997. Dynamics of adaptation and evolutionary branching. Physical Review Letters 78, 20242027.

Hamilton, W. D., 1967. Extraordinary sex ratios. Science 156, 477-488.

Heino, M., Metz, J. A. J., Kaitala, V., 1997. Evolution of mixed maturation strategies in semelparous life-histories: the crucial role of dimensionality of feedback environment. Proceedings of the Royal Society London, B 352, 
$1647-1655$.

Heino, M., Metz, J. A. J., Kaitala, V., 1998. The enigma of frequencydependent selection. Trends in Ecology and Evolution 13, 367-370.

Hofbauer, J., Sigmund, K., 1990. Adaptive dynamics and evolutionary stability. Applied Mathematical Letters 3, 75-79.

Huisman, J., Weissing, F. J., 1999. Biodiversity of plankton by species oscillations and chaos. Nature 402, 407-410.

Kawecki, T. J., 1993. Age and size at maturation in a patchy environment: fitness maximization versus evolutionary stability. Oikos 66, 309-317.

Kisdi, É., Meszéna, G., 1993. Density dependent life history evolution in fluctuating environments. In: Yoshimura, J., Clark, C. W. (Eds.), Adaptations in Stochastic Environments. Lecture Notes in Biomathematics 98, Springer, Berlin.

Kisdi, É., Jacobs, F. J. A., Geritz, S. A. H., 2000. Red Queen Evolution by Cycles of Evolutionary Branching and Extinction. Selection 2: 161-176.

Koch, A. L., 1974. Competitive coexistance of two predators utilizing the same prey under constant environmental conditions. Journal of Theoretical Biology 44, 387-395.

Lande, R., 1976. Natural selection and random genetic drift in phenotypic evolution. Evolution 30, 314-334.

Lawlor, L. R., Maynard Smith, J., 1976. The coevolution and stability of competing species. The American Naturalist 110, 79-99.

Leimar, O., 2001. Evolutionary change and darwinian demons. Selection 2, $65-72$.

Lessells, C. M., 1991. Behavioural Ecology, 3rd Edition. Blackwell Scientific Publications, Ch. The Evolution of Life Histories, pp. 32-68.

Levins, R., 1962. Theory of fitness in a heterogeneous environment. 1. the 
fitness set and the adaptive function. The American Naturalist 96, 361373.

Levins, R., 1968. Evolution in changing environments. Vol. 2 of Monographs in Population Biology. Princeton University Press, Princeton, New Jersey.

Levins, R., 1979. Coexistence in a variable environment. The American Naturalist 114, 765-783.

Marrow, P., Law, R., Cannings, C., 1992. The coevolution of predator-prey interactions: ESS and red queen dynamics. Proceedings of the Royal Society London, B 250, 133-141.

Matessi, C., Di Pasquale, C., 1996. Long-term evolution of multilocus traits. Journal of Mathematical Biology 34, 613-653.

Mathias, A., Kisdi, É., 1999. Evolutionary Branching and Coexistence of Germination Strategies. IIASA Interim Report IR-99-014, available at http://www.iiasa.ac.at/Research/ADN/Series.html.

Mathias, A., Kisdi, É., 2002. Adaptive diversification of germination strategies. Proceedings of the Royal Society London, B 269, 151-155.

Matsuda, H., 1985. Evolutionary stable strategies for predator switching. Journal of Theoretical Biology 115, 351-366.

Maynard Smith, J., 1978. Optimization theory in evolution. Annual Review in Ecology in Systematics 9, 31-56.

Maynard Smith, J., 1982. Evolution and the Theory of Games. Cambridge University Press, Cambridge, U. K.

Maynard Smith, J., Price, G. R., 1973. The logic of animal conflict. Nature $246,15-18$.

McNamara, J. M., 1995. Implicit frequency-dependence and kin selection in fluctuating environments. Evolutionary Ecology 9, 185-203.

McNamara, J. M., Houston, A. I., Collins, E. J., 2001. Optimality models in 
behavioral biology. SIAM Review 43, 413-466.

Meszéna, G., Kisdi, É., Dieckmann, U., Geritz, S. A. H., Metz, J. A. J., 2001. Evolutionary optimization models and matrix games in the unified perspective of adaptive dynamics. Selection 2, 193-210.

Metz, J. A. J., Geritz, S. A. H., Meszéna, G., Jacobs, F. J. A., Van Heerwaarden, H. J., 1996a. Adaptive dynamics: A geometrical study of the consequences of nearly faithful reproduction. In: van Strien, S. J., Verduyn Lunel, S. (Eds.), Stochastic and spatial structures of dynamical systems. Proceedings of the Royal Dutch Academey of Science, North Holland, Elsevier, pp. $183-231$.

Metz, J. A. J., Mylius, S. D., Diekmann, O., 1996b. When does evolution optimize? On the relation between types of density dependence and evolutionarily stable life history parameters. IIASA Working Paper WP-96-04, available at http://www.iiasa.ac.at/Research/ADN/Series.html.

Metz, J. A. J., Nisbet, R. M., Geritz, S. A. H., 1992. How should we define 'fitness' for general ecological scenarios? Trends in Ecology and Evolution $7,198-202$.

Michod, R. E., 1979. Evolution of life histories in response to age-specific mortality factors. The American Naturalist 113, 531-550.

Motro, U., 1982. Optimal Rates of Dispersal I. Haploid Populations. Theoretical Population Biology 21, 394-411.

Motro, U., 1994. Evolutionary and continuous stability in asymmetric games with continuous strategy sets: the parental investment conflict an an example. The American Naturalist 144, 229-241.

Mylius, S. D., Diekmann, O., 1995. On evolutionary stable life histories, optimization and the need to be specific about density dependence. Oikos 74, 218-224. 
Nowak, M., 1990. An evolutionarily stable strategy may be inaccessible. Journal of Theoretical Biology 142, 237-241.

Philippi, T., Seger, J., 1989. Hedging one's evolutionary bets revisited. Trends in Ecology and Evolution 4, 41-44.

Pianka, E. R., Parker, W. S., 1975. Age-specific reproductive tactics. The American Naturalist 109, 45-464.

Reed, J., Stenseth, N. C., 1984. On evolutionary stable strategies. Journal of Theoretical Biology 108, 491-508.

Roff, D., 1992. The evolution of life histories. Chapman \& Hall, New York.

Roff, D., 2002. Life history evolution. Sinauer, Sunderland, Massachusetts, USA.

Schaffer, W. M., 1974. Selection for optimal life histories, the effects of age structure. Ecology 55, 291-303.

Schluter, D., 1993. Adaptive radiation in sticklebacks: Size, shape, and habitat use efficiency. Ecology 74, 699-709.

Stearns, S. C., 1992. The evolution of life histories. Oxford University Press, Oxford, UK.

Stearns, S. C., 2000. Life history evolution: successes, limitations, and prospects. Naturwissenschaften 87, 476-486.

Stephens, D. W., Krebs, J. R., 1986. Foraging Theory. Princeton University Press, Princeton, NJ.

Svensson, E., Sheldon, B. C., 1998. The social context of life history evolution. Oikos 83, 466-477.

Takada, T., 1995. Evolution of semelparous and iteroparous perennial plants: Comparison between the density-independent and the density-dependent dynamics. Journal of Theoretical Biology 173, 51-60.

Taylor, P. D., 1989. Evolutionary stability of one-parameter models under 
weak selection. Theoretical Population Biology 36, 125-143.

van Tienderen, P. H., de Jong, G., 1986. Sex ratio under the haystack model: Polymorphism may occur. Journal of Theoretical Biology 122, 69-81.

Vincent, T. L., Brown, J. S., 1988. The evolution of ess theory. Annual Review of Ecology and Systematics 19, 423-443.

Wilson, D. S., Yoshimura, J., 1994. On the coexistence of specialists and generalsits. The American Naturalist 144, 692-707.

Wright, S., 1931. Evolution in mendelian populations. Genetics 16, 97-159.

Yodzis, P., 1989. Introduction to Theoretical Ecology. Harper \& Row, NY. 
Table 1

Classification of the evolutionarily singular strategies $\theta^{*}$ with respect to (1) the relative curvature of the trade-off curve and the invasion boundary and (2) the difference in slope the between trade-off curve and the invasion boundary.

\begin{tabular}{|c||c|c|}
\hline \multicolumn{1}{|c||}{} & Noninvadable & Invadable \\
& $\frac{\partial(\mu-\nu)}{\partial \phi}\left(\theta^{*}\right)<0$ & $\frac{\partial(\mu-\nu)}{\partial \phi}\left(\theta^{*}\right)>0$ \\
\hline \hline Convergence stable & & \\
$\frac{\partial(\mu-\nu)}{\partial \theta_{r}}\left(\theta^{*}\right)<0$ & Continuously Stable Strategy & Branching Point \\
\hline Repelling & Garden of Eden & Repellor \\
$\frac{\partial(\mu-\nu)}{\partial \theta_{r}}\left(\theta^{*}\right)>0$ & & \\
\hline
\end{tabular}


Figure 1. Fixed fitness contour lines in a two-dimensional trait space plotted on top of a concave (a) and a convex (b) trade-off curve. Under the assumption that fitness is increasing in both traits, the open circles correspond to strategies that maximize fitness. For the concave trade-off (a) this happens at the point where the fitness contour line is tangent to the trade-off curve. For the convex trade-off (b) this happens at either of the two boundary strategies.

Figure 2. Invasion boundaries in a two-dimensional trait space for strategies lying one the trade-off curve (traits named $\phi$ and $\psi$, see text). When invasion boundaries are linear, CSSs and Garden of Eden points are only possible in combination with concave trade-offs (a, c), while Repellors and Branching Points can only occur on convex trade-offs (b, d). Resident strategies are indicated by an open circle and the arrows indicate the direction of further trait substitutions.

Figure 3. Invasion boundaries in a two-dimensional trait space for strategies lying one the trade-off curve (traits named $\phi$ and $\psi$, see text). If invasion boundaries are not bound to be linear, each of the four evolutionarily singular points can occur in combination with both convex and concave trade-off curves.

Figure 4. Branching point in the trait $\theta$ : (a) Represented as a pairwise invasibility plot (PIP), resident trait value at the $x$-axis and mutant trait value at the $y$-axis. (b-d) Fitness landscape representation for three different resident strategies $\theta_{r}$ (1-3) which lie on the trade-off curve. Resident strategies are indicated by an open circle. Mutants which are selectively neutral and therefore lie on the invasion boundary are indicated by a filled circle. Arrows indicate the direction of the evolutionary dynamics along the trade-off curve. The plus sign indicates mutant strategies that are able to invade.

Figure 5. Dimorphic evolution on a convex trade-off curve with a linear invasion boundary. The resident types $\theta_{1}$ and $\theta_{2}$ are indicated by open circles. Arrows indicate the direction of selection corresponding to the resident strategies. 
Figure 6. Scenario of dimorphic evolution where one resident type $\left(\theta_{2}\right)$ goes extinct in the course of evolution (indicated by a circle with a dotted boundary) and the other type $\left(\theta_{1}\right)$ remains. The process is sketched when the evolutionary trajectory changes from dimorphic to monomorphic. At this moment selection favors further specialization of the remaining type (indicated by arrow). This scenario is only possible with a Repellor (Rep) between the Branching Point (BP), where the polymorphism emerged, and the remaining type. See text for further details. 
Figure (1)
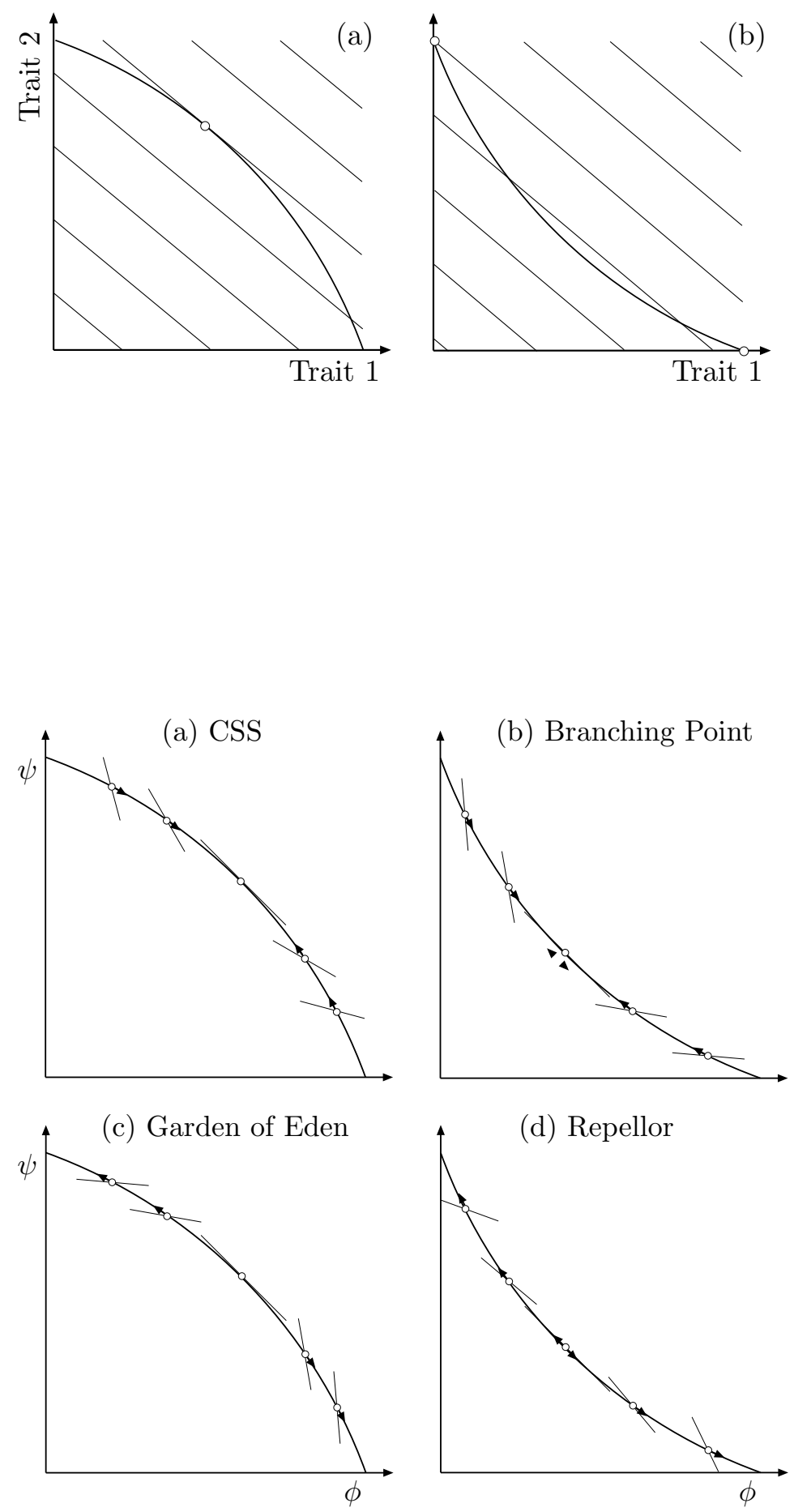

Figure (2) 


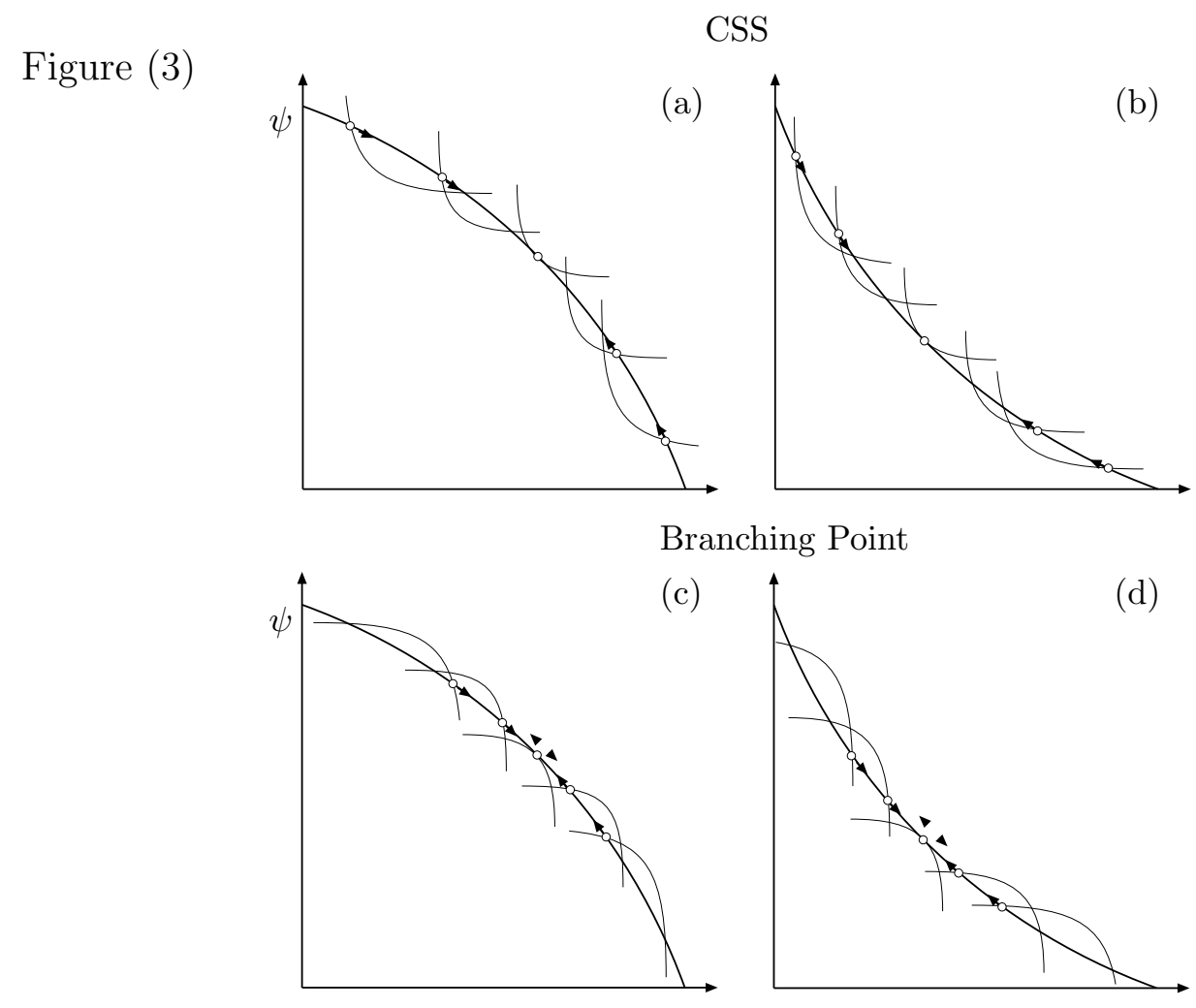

Garden of Eden


Repellor

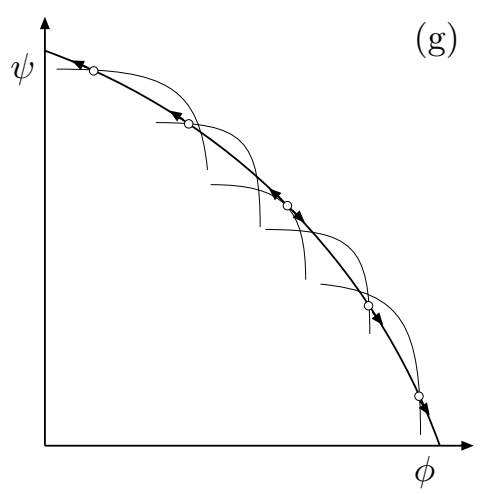

(h)

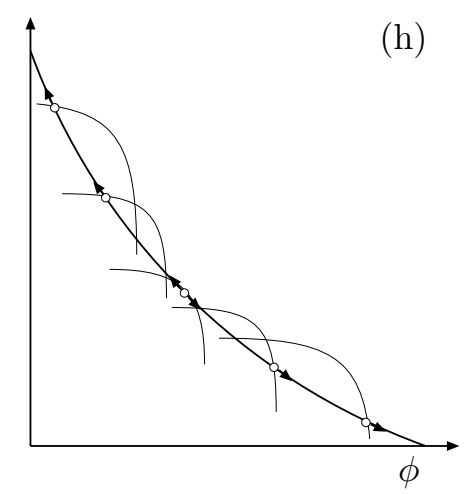


Figure (4)
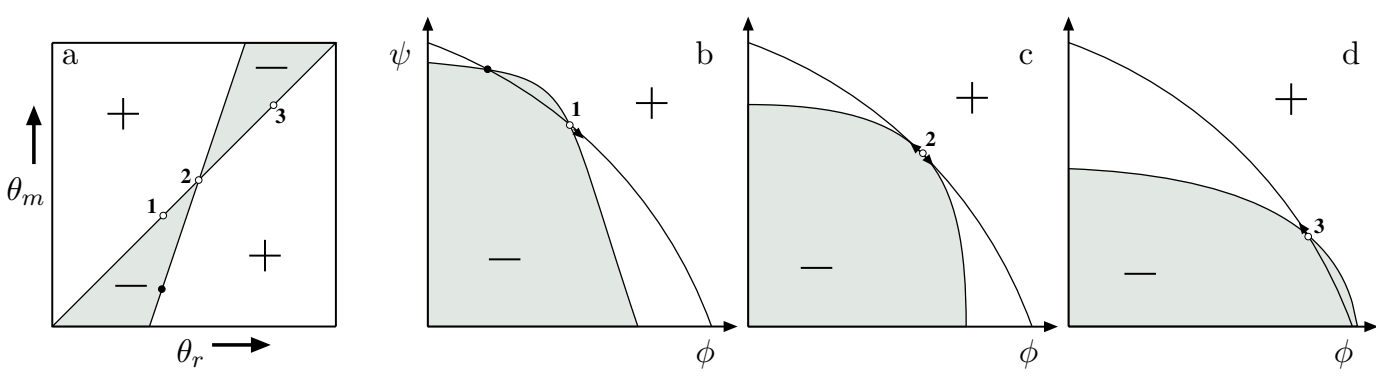

Figure (5)

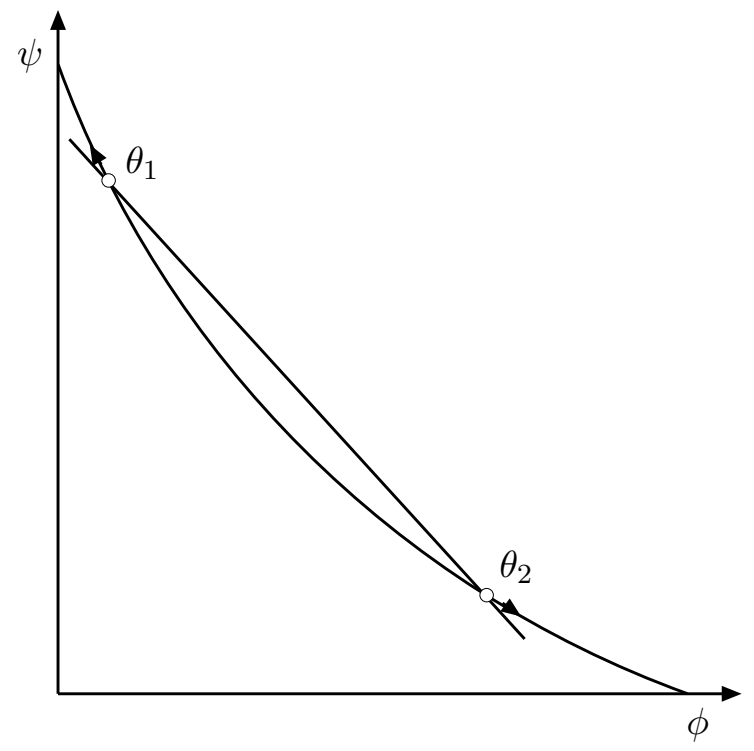

Figure (6)

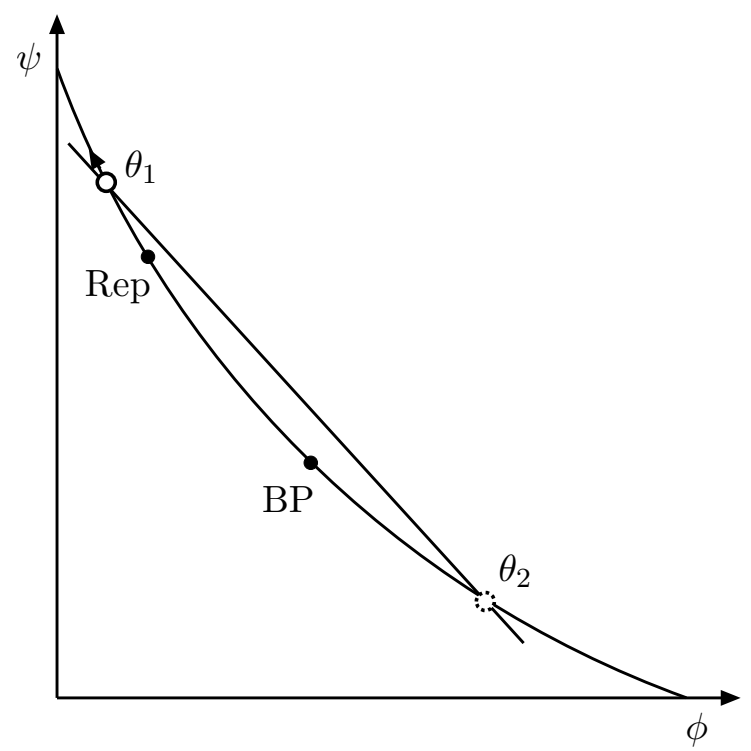

